\title{
A PESQUISA NA ÁREA dO VAREJO: REFLEXÕES E PROVOCAÇÕES
}

Eliane Pereira Zamith Brito eliane.brito@fgv.br

Professora da FGV-EAESP

Valter Afonso Vieira valterafonsovieira@yahoo.com

Professor do PPA/UEM

Lélis Balestrin Espartel lbespartel@pucrs.br

Professor do PPGAd/PUC-RS

\section{INTRODUÇÃO}

Uma análise criteriosa da recente produção científica no campo do varejo aponta deficiências ou alguns "esquecimentos acadêmicos". A produção acadêmica em varejo encontra-se dispersa em uma enorme quantidade de periódicos nacionais e internacionais. Entre eles, sem dúvida alguma, o de maior impacto é o Journal of Retailing (JCR $2011=2,25)$, considerado $\mathrm{O}$ periódico mais longevo dentro do marketing, tendo sido estabelecido em 1925. Segundo informações editoriais do Journal of Retailing, o "campo do varejo inclui produtos e serviços, a cadeia de suprimentos e os canais de distribuição que servem aos varejistas, os relacionamentos entre varejistas e membros do canal, e todas as formas de marketing direto e mercados eletrônicos emergentes até os domicílios".

Outros dois periódicos específicos da área de varejo merecem destaque. O International Journal of Retail \& Distribution Management (anteriormente publicado como Retail and Distribution Management) tem como foco, de acordo com a linha editorial, a questão de "significância estratégica em varejo e distribuição" e "proporciona uma ligação entre a produção e o consumidor".

Já o Journal of Retailing and Consumer Services tem seu foco "particularmente no comportamento do consumidor e em decisões políticas e gerenciais" atinentes ao varejo, cobrindo distribuição de bens, varejo de serviços profissionais (p. ex., saúde) e varejo de serviços ao consumidor (p. ex., turismo).

Não existe no Brasil um periódico cuja política editorial esteja voltada exclusivamente para temas relacionados ao varejo e, por isso, é difícil contabilizar a produção nacional nessa área.

Há uma nítida lacuna entre o que os periódicos internacionais de marketing se propõem a fomentar, do ponto de vista de contribuição empírica, e o que se verifica de maneira efetiva nos volumes editados. Por exemplo, o Journal of Retailing, em sua linha editorial, busca publicar trabalhos sobre a 
relação B2B na cadeia de suprimento, marketing direto no varejo, consumo familiar em mercados emergentes, modelos matemáticos e de previsões, técnicas estatísticas avançadas no varejo, experimentos de campo, impacto da tecnologia na prestação do serviço e, inclusive, comportamento do consumidor. Não obstante, nota-se claramente uma ênfase demasiada em artigos relacionados ao comportamento do consumidor em detrimento de outros, notadamente de mesma relevância (GREWAL e LEVY, 2007).

Levy e Weitz (2004, p. 6) definem varejo como "um conjunto de atividades de negócio que adicionam valor aos produtos e serviços vendidos aos consumidores para seu uso pessoal ou familiar". Como decorrência, compreende-se o varejo como uma atividade econômica, e, como tal, deveria tratar de operações, finanças, estratégia, internacionalização, pessoas, inteligência de mercado, marketing, além de geografia, engenharia, meio ambiente, entre outros aspectos. Em vez de uma solução interdisciplinar aos problemas varejistas, há uma superespecialização na relação entre o varejista e o consumidor, diminuindo também a amplitude da relevância gerencial, uma vez que representa apenas um extremo da cadeia, o comportamento do consumidor.

\section{PANORAMA INTERNACIONAL DA PESQUISA NO VAREJO}

A pesquisa no campo do varejo é ampla, profunda e rica, sendo um dos campos mais férteis dentro do marketing (GREWAL, LEVY,
KUMAR, 2009). Não obstante, O varejo tem experimentado mudanças significativas nos últimos anos (GREWAL e LEVY, 2007), passando de varejo físico para virtual, para citar um dos tantos exemplos disponíveis.

Enquanto alguns temas de pesquisa na área de varejo têm despertado maior interesse dos pesquisadores e gestores, e, por consequência, gerado um volume maior de contribuições científicas para a comunidade empresarial, outros permanecem sem destaque ou perderam a relevância de outrora.

De acordo com Grewal e Levy (2007), então editores do Journal of Retailing, um levantamento realizado no período de seis anos, entre 2002 e 2007, indica a publicação de 164 artigos, sendo 130 regulares, 20 em edições especiais, cinco convidados e nove editoriais. Desse total, os autores agruparam os artigos de acordo com as principais contribuições de cada um em 10 grandes categorias, permitindo que um mesmo artigo fosse classificado em múltiplas categorias. Diante disso, a Tabela 1 mostra as principais áreas dos trabalhos publicados, salientando a linha de pesquisa no campo.

Os dados mostram uma grande quantidade de trabalhos publicados no campo do comportamento do consumidor e da lealdade do cliente, somando um total aproximado de 30\% dos trabalhos. Algumas possíveis explicações para essa abundância de trabalhos dentro da linha podem advir do fato de que: ( $i$ ) muitos artigos classificados em outras categorias, tais como preço, propaganda e internet, por exemplo, tratam igualmente de comportamento do consumidor - tal aspecto dá mais ênfase no respondente comprador do que na gestão de marketing; (ii) após a Segunda Guerra Mundial, houve um aumento importante da natalidade nos Estados Unidos, criando a geração baby boom, a qual ocasionou um impacto significativo no consumo e passou a ser objeto de estudo; (iii) houve um grande aumento na quantidade de periódicos disponíveis para publicação dos artigos em comportamento do consumidor; (iv) existiu um grande aumento nas escolas de administração que possuem doutoramento, laboratórios e linhas de pesquisa

\begin{tabular}{|c|c|c|}
\hline ÁREA & $\mathrm{N}^{0}$ DE ARTIGOS & $\%$ \\
\hline Comportamento do consumidor & 46 & 18,5 \\
\hline Preço & 39 & 15,7 \\
\hline Lealdade & 30 & 12,1 \\
\hline Serviços & 26 & 10,5 \\
\hline Internet & 23 & 9,3 \\
\hline Produto/Marca & 17 & 6,9 \\
\hline Organizações & 15 & 6,0 \\
\hline Promoções & 19 & 7,7 \\
\hline Canal & 14 & 5,6 \\
\hline Outros (p. ex., ética, varejo global, formato) & 19 & 7,7 \\
\hline
\end{tabular}

Fonte: adaptado de Grewal e Levy, 2007, e Brown e Dant, 2008. 
exclusivas na área de comportamento do consumidor e (iv) houve crescimento e utilização da psicologia aplicada ao comportamento do consumidor (por ex., experimental, humanista, social, cognitiva, sociocultural etc.). Adiante, apresentar-se-ão áreas de pesquisa que são relevantes para o campo do varejo e que poderiam ampliar o escopo da pesquisa na área.

\section{NOVAS TRILHAS PARA PESQUISAS FUTURAS}

Como ampliar o escopo de estudo no varejo, abrangendo temas mais relevantes a toda a cadeia de suprimentos? Como estimular pesquisadores a desenvolverem seus projetos de pesquisa de modo a contemplar outras relações ou contextos na cadeia que não exclusivamente a pesquisa com os consumidores finais? Objetivando buscar respostas para tais questões, alguns tópicos para pesquisas futuras e avanços no varejo são apresentados a seguir.

O acompanhamento da produção acadêmica em varejo na última década revela algumas tendências e temas emergentes em varejo. De acordo com Grewal e Levy (2009), alguns temas podem ser vistos como emergentes e podem ser agrupados da seguinte forma:

- Aumento do papel do cliente: aqui vale ressaltar o espaço destinado para discussão da interatividade do consumidor com o varejo eletrônico (ZEITHAML, PARASURAMAN, MALHOTRA, 2002; YADAV e VARADARAJAN, 2005), pesquisando pontos como a forma pela qual o consumidor pode interagir com o varejo no sentido de criar produtos, serviços ou processos. A Skol Beats 2008, por exemplo, lançou essa ideia com a "mão amarela";

- Experiência de compra: há também os estudos e movimentos gerenciais varejistas no sentido de transformar a compra de produtos ou serviços no varejo em experiências de compra (VERHOEF e outros, 2009), sobretudo em uma experiência social (BORGES, CHEBAT, BABIN, 2010). A loja conceito da Heineken Experience, em Amsterdã, a Nike ID Studio, localizada em Nova Iorque, e a loja Mi Adidas Innovation Center, localizada em Paris, Champs-Elysées, são ilustrações da experiência de compra;

- Novas tecnologias: há necessidade de novas pesquisas na análise do impacto de novas tecnologias no desempenho varejista, tais como o mobile marketing e aplicativos em realidade aumentada no varejo, facilitando a compra de produto (RYDING, 2010). O banco Bradesco recentemente lançou uma nova tecnologia de internet banking para o banco mobile;

- Imagem da marca: neste ponto, pode-se discutir os já disseminados cartões de fidelidade e suas implicações para o varejo e para a imagem da marca do varejista (DEMOULIN e ZIDDA, 2009). O McDonald's, por exemplo, criou um programa de bonificação especial para clientes que compram unicamente via drive-thru;

- Estratégias de serviço: tradicionalmente, este é um tema explorado no varejo (GREWAL e LEVY, 2009), havendo espaço para análise de temas como tecnologia de autosserviço (por ex., caixas nos bancos de varejo), gestão da compra em múltiplos canais (por ex., telefone, internet, correio etc.), recuperação de serviços, entre outros (GREWAL e LEVY, 2007). A ênfase na estratégia de serviço no varejo é consistente com as proposições de Vargo e Lusch (2004) e Lusch, Vargo e O’Brien (2007);

- Compras coletivas: este conceito de compra faz com que novas empresas façam a intermediação entre o varejista e o comprador, alavancando a quantidade de vendas (CLARK, 2001). O volume das compras coletivas em 2010, no Brasil, foi de $\mathrm{R} \$ 10,7$ milhões e continua crescendo (Notícias, 2011);

- Mídias sociais: são um canal relevante para a empresa comunicar promoções, apresentar programas de responsabilidade social, interagir com o cliente e se relacionar com os fornecedores. Tais pontos ainda necessitam de estudos para compreender melhor seus impactos na organização e na sociedade;

- Franquias: a temática de franquia toma cada vez mais corpo, e ganhou evidência desde que a franquia Subway ultrapassou o McDonald's em número de lojas ao redor do mundo. Temas como a associação de franquias (que objetiva defender o franqueado), modelos de contrato em franquias, programas de expansão global (caso do Boticário), entre outros, são de potencial interesse de pesquisa;

- Estratégia varejista: muito se 
comenta sobre fusão, aquisição e expansão no varejo. Tal temática é extremamente interessante do ponto de vista organizacional, econômico, financeiro e de mercado. O negócio firmado entre o Grupo Pão de Açúcar e as Casas Bahia foi baseado na integração dos negócios do setor do varejo de bens duráveis e, após a fusão, o grupo tem mais de 1.015 lojas, com faturamento de $\mathrm{R} \$ 18,1$ bilhões e $68 \mathrm{mil}$ funcionários;

- Relacionamento no canal: o campo do varejo carece de trabalhos que investiguem $\mathrm{O}$ relacionamento no canal B2B. Questões sobre comportamentos oportunistas entre fornecedor e fabricante, nível de comunicação dentro do canal e intenção de permanecer no canal são interessantes e podem apresentar novas formas de trabalho no relacionamento com o cliente industrial (VIEIRA, MONTEIRO, VEIGA, 2011);

- Ambiente de loja: percebe-se também espaço para estudos aprofundados a respeito da ambientação de loja, originalmente propostos por Kotler (1973), com substanciais contribuições da Teoria da Psicologia Ambiental, de Mehrabian e Russel (1974), e que encontrou eco em estudos seminais, como os de Bitner (1992), de Baker e outros (2002) e de Turley e Chebat (2002);

- Reduções de embalagem: afetam a escolha do consumidor, a disposição e os espaços nas gôndolas, a economia do fabricante, entre outros fatores. Pesquisar tal vertente é de extrema relevância para o setor (VIEIRA, 2003);
- Uso de datamining: aplicação de mineração de dados para envio de malas diretas, definição de padrão de compra, elaboração de promoções, entre outros.

- Estímulos de loja: na literatura de varejo, existe considerável corpo teórico que analisa o impacto dos estímulos salientados pelo ambiente sobre as emoções e sobre as respostas dos compradores. Essa sequência é compreendida como modelo "E-O-R". Pela perspectiva preditora dos estímulos, alguns trabalhos verificaram que a música (MATTILA e WIRTZ, 2001; MILLIMAN, 1982), O cheiro (BONÉ e ELLEN, 1999) e o ambiente (SHERMAN, MATHUR, SMITH, 1997) influenciam as emoções dos clientes. Por outro lado, as emoções dos consumidores impactam nas respostas, compreendidas como os gastos na loja (BABIN e DARDEN, 1995), o volume de vendas (MILLIMAN, 1982), o tempo no local (SHERMAN, MATHUR, SMITH, 1997) e a tendência de procurar mais produtos (MENON e KAHN, 2002);

- Precificação: conforme a Tabela 1, preço já é o segundo tema de interesse em pesquisas no Journal of Retailing. Conforme Grewal e Levy (2009), ainda existem possibilidades de pesquisa em preços, como possíveis conflitos entre táticas de preço e estratégias de precificação, pacotes de preço e respostas dos clientes às mudanças nos preços. Há também a área do estabelecimento de preços no varejo para produtos de informação. Esses produtos são normalmente intangíveis e com processo de distribuição eletrônica, os quais estão cada vez mais presentes no mercado, possuindo características distintas do processo de precificação dos produtos físicos, cuja distribuição é mais fácil de controlar.

\section{FÓRUM VAREJO}

A importância da atividade varejista no Brasil e o aumento da competição nessa atividade, fundamentado nas capacidades da empresa, fizeram com que os pesquisadores do Centro de Excelência em Varejo da Escola de Administração de Empresas de São Paulo da Fundação Getulio Vargas (CEV/EAESP/ FGV) vislumbrassem, em 2008, a possibilidade de criar o Congresso Latino-Americano de Varejo (CLAV). Tal iniciativa tem como objetivo encontrar respostas eficientes para problemas existentes na arena da gestão empresarial e tem servido como um fórum para pesquisadores e gestores criarem e apresentarem soluções, ferramentas e modelos confiáveis, válidos e robustos para melhorar a atuação das organizações.

Após três anos de prazeroso e cauteloso trabalho, o CLAV se solidifica no cenário nacional e lança o primeiro Fórum de Varejo na RAE-Revista de Administração de Empresas, contendo trabalhos apresentados no congresso. Foram sete trabalhos submetidos a avaliação double blind por um conjunto de avaliadores ad hoc com experiência e interesse de pesquisa em marketing e varejo. Desses artigos, foram aprovados cinco; o tempo médio que os artigos ficaram em avaliação foi 239 dias, conside- 
rando que, entre os cinco artigos publicados, três foram aprovados na segunda versão e dois na terceira versão, os quais comentamos brevemente a seguir.

Em "O aroma ambiental e sua relação com as avaliações e intenções do consumidor no varejo", André Luiz Carvalho Nunes da Costa e Salomão Alencar de Farias retomam a questão da atmosfera de loja de varejo, especificamente estudando o aroma ambiental, explorando a relação entre os estímulos olfativos e as respostas dos consumidores. A realização de um quase experimento em uma butique de frutos do mar indicou que a manipulação do aroma não trouxe impactos nas avaliações do consumidor a respeito da loja, tampouco em sua intenção de retorno, mas impactou positivamente no tempo de permanência dentro da loja.

Os pesquisadores Christiano França da Cunha, Eduardo Eugênio Spers e Decio Zylbersztajn, no artigo "Percepção sobre atributos de sustentabilidade em um varejo supermercadista", discutem os selos e certificados como forma de o consumidor monitorar e avaliar a qualidade dos alimentos. Com base em uma survey com 120 consumidores, os resultados indicam quatro dimensões relevantes, sendo: segurança alimentar, qualidade intrínseca do produto, sistema de produção diferente e verificação do governo. Além disso, valores como prestígio, credibilidade e respeito foram considerados relevantes em relação à sustentabilidade e à origem.

No artigo "Avaliação de justiça e intenção de turnover em equipes de vendas: teste de um modelo teórico", Evandro Luiz Lopes, Sérgio Luiz do Amaral Moretti e Thomas Brashear Alejandro investigam as relações entre justiça e rotatividade de empregados. Baseados em uma survey com 194 profissionais de vendas, os autores propõem uma nova escala para mensuração de justiça percebida e aprofundam a discussão sobre a multidimensionalidade da percepção de justiça, lealdade, motivação e da intenção de turnover.

Abrão Caro, José Afonso Mazzon, Barbara Caemmerer e Matthias Wessling apresentam "Inovatividade, envolvimento, atitude e experiência na adoção da compra on-line". No artigo, os autores discutem as mudanças nos hábitos e nos estilos de compra decorrentes das diferenças entre lojas virtuais e lojas físicas. A análise do modelo integrativo proposto aponta que a intenção da compra pela internet é diretamente influenciada pela atitude e pela inovatividade, e a atitude é influenciada pelo envolvimento. Entretanto, não foi encontrada relação entre a experiência com a internet e a compra pela internet.

No último artigo desta edição, "Conveniência de serviços: apropriação e adaptação de uma escala de medida”, Edar da Silva Añaña, Renata Gonçalves Santos Silva e Walter Meucci Nique propõem a adequação da Escala de Conveniência de Serviços - Servcon para uso na realidade brasileira, mais especificamente no varejo de livre localização das cidades de porte médio. Após a aplicação de dois levantamentos, os resultados indicaram que, para adequação ao contexto nacional, é necessária a inserção de fatores relacionados à segurança, à mobilidade e ao regramento da informalidade.

Este volume da $R A E$ apresenta cinco artigos que, de uma forma geral, representam, para o campo do varejo, mesmo que seja apenas uma pequena contribuição para a desejada ampliação do escopo da pesquisa na área, a posição defendida no início desta apresentação. Assim, tem-se aqui uma mescla de trabalhos, de áreas, de métodos e de contribuições para a gestão varejista que abordam pontos como aroma de loja, atributos de segurança no varejo, justiça na equipe de vendas, adoção de compra pela internet e métrica de conveniência no varejo. Elaborar uma edição especial no campo do varejo é uma tarefa conjunta de diversos profissionais. Portanto, os editores são extremamente gratos pela contribuição anônima dos revisores, da secretaria da $R A E$ e do editor.

\section{REFERÊNCIAS}

BABIN, B. J; DARDEN, W. R. Consumer self-regulation in a retail environment. Journal of Retailing, v. 71, n. 1, p. 47-71, 1995.

BAKER, J. e outros. The influence of multiple store environment cues on perceived merchandise value and patronage intentions. Journal of Marketing, v. 66, n. 2, p. 120-141, 2002.

BITNER, M. J. Servicescapes: the impact of physical surroundings on customers and employees. Journal of Marketing, v. 56, n. 2, p. 57-71, 1992.

BONÉ, P. F; ELLEN, P. S. Scents in the marketplace: explaining a fraction of olfaction. Journal of Retailing, v. 75 , n. 2, p. 243-262, 1999.

BORGES, A; CHEBAT, J. C; BABIN, B. $J$. Does a companion always enhance the shopping experience? Journal of Retailing and Consumer Services, v. 17, n. 3, p. 294-299, 2010. 
BROWN, J. R; DANT, R. P. Scientific method and retailing research: a retrospective. Journal of Retailing, v. 84, n. 1, p. 1-13, 2008.

CLARK, D. Mobshop, a pioneer in group-buying on the Web, discontinues consumer service. Wall Street Journal, 2001. Disponível em: www. mobshop.com/ar011501d.

DEMOULIN, N. T. M; ZIDDA, P. Drivers of customers' adoption and adoption timing of a new loyalty card in the grocery retail market. Journal of Retailing, v. 85, n. 3, p. 391-405, 2009.

GREWAL, D; LEVY, M. Emerging issues in retailing research. Journal of Retailing, v. 85, n. 4, p. 522-526, 2009.

GREWAL, D; LEVY, M. Retailing research: past, present, and future. Journal of Retailing, v. 83, n. 4, p. 447-464, 2007.

GREWAL, D; LEVY, M; KUMAR, V. Customer experience management in retailing: an organizing framework. Journal of Retailing, v. 85, n. 1, p. 1-14, 2009.

KOTLER, P. Atmospherics as a marketing tool. Journal of Retailing, v. 49, n. 4, p. 48-64, 1973.

LEVY, M; WEITZ, B. A. Retailing management. Boston: McGraw-Hill, 2004.

LUSCH, R. F; VARGO, S. L; O'BRIEN, $M$. Competing through service: insights from service-dominant logic. Journal of Retailing, v. 83, n. 1, p. 2-18, 2007.

MATTILA, A. S; WIRTZ, J. Congruency of scent and music as a driver of in-store evaluations and behavior. Journal of Retailing, v. 77, n. 2, p. 273289, 2001.
MEHRABIAN, A; RUSSEL, J. A. An approach to environmental psychology. Cambridge: MIT Press, 1974.

MENON, S; KAHN, B. Cross-category effects of induced arousal and pleasure on the internet shopping experience. Journal of Retailing, v. 78, n. 1, p. 31-40, 2002.

MILLIMAN, R. E. Using background music to affect the behavior of supermarket shoppers. Journal of Marketing, v. 46, n. 3, p. 86-91, 1982.

NOTÍCIAS. Sites de compras coletivas movimentam $\mathrm{R} \$ 10,7$ milhões em 2010. 2011. Disponível em: http://portalwwww.d24am.com/noticias/econo$\mathrm{mia} /$ sites-de-compras-coletivas-movimentam-r-107-milhes-em-2010/27946. Acesso em 20.10.2011.

RYDING, D. The impact of new technologies on customer satisfaction and business to business customer relationships: evidence from the soft drinks industry. Journal of Retailing and Consumer Services, v. 17, n. 4, p. 224-228, 2010.

SHERMAN, E; MATHUR, A; SMITH, R. B. Store environment and consumer purchase behavior: mediating role of consumer emotions. Psycho$\log y$ \& Marketing, v. 14, n. 4, p. 361378, 1997.

TURLEY, L. W; CHEBAT, J. C. Linking retail strategy, atmospheric design and shopping behaviour. Journal of Marketing Management, v. 18, n. 2, p. 125-144, 2002.

VARGO, S. L; LUSCH, R. F. Evolving to a new dominant logic for marketing. Journal of Marketing, v. 68, n. 1, p. 1-17, 2004.
VERHOEF, P. C. e outros. Customer experience creation: determinants, dynamics and management strategies. Journal of Retailing, v. 85, n. 1 , p. 31-41, 2009.

VIEIRA, V. A; MONTEIRO, P. R. R; VEIGA, R. T. Relationship marketing in supply chain: an empirical analysis in the Brazilian service sector. Journal of Business \& Industrial Marketing, v. 26, n. 7, p. 524-531, 2011.

VIEIRA, F. G. D. A soberania do consumidor como um mito perante situações de redução de embalagens no mercado brasileiro. In: ENCONTRO ANUAL DA ASSOCIAÇÃO NACIONAL DOS PROGRAMAS DE PÓS-GRADUAÇÃO EM ADMINISTRAÇÃO, 27, 2003 , Atibaia. Anais do EnANPAD 2003, Área de Marketing. Rio de Janeiro: Associação Nacional dos Programas de Pós-Graduação em Administração, 2003. v. 1. p. 1-16.

YADAV, M. S; VARADARAJAN, P. R. Interactivity in the electronic marketplace: an exposition of the concept and implications for research. Journal of the Academy of Marketing Science, v. 33, n. 4, p. 585-603, 2005.

ZEITHAML, V. A; PARASURAMAN, A; MALHOTRA, A. Service quality delivery through Web sites: a critical review of extant knowledge. Journal of the Academy of Marketing Science, v. 30, n. 4, p. 362-375, 2002. 